PROCEEDINGS OF THE

AMERICAN MATHEMATICAL SOCIETY

Volume 135, Number 5, May 2007, Pages 1511-1514

S 0002-9939(06)08819-8

Article electronically published on November 29, 2006

\title{
ON UNIFORMLY GÂTEAUX SMOOTH NORMS AND NORMAL STRUCTURE
}

\author{
MICHAL JOHANIS AND JAN RYCHTÁR̆
}

(Communicated by Jonathan M. Borwein)

\begin{abstract}
It is shown that every separable Banach space admits an equivalent norm that is uniformly Gâteaux smooth and yet lacks asymptotic normal structure.
\end{abstract}

A Banach space is said to have the fixed point property (FPP) if for every nonempty bounded closed convex $C \subset X$ and every nonexpansive self-mapping $T: C \rightarrow C$ there is a fixed point of $T$ in $C$. A Banach space is said to have the weak fixed point property (w-FPP) if for every nonempty weakly compact convex $C \subset X$ there is a fixed point for every nonexpansive $T: C \rightarrow C$. Clearly, a Banach space has w-FPP if it has FPP. The space $c_{0}$ has w-FPP but does not have FPP; see $[\mathrm{M}$. These two notions obviously coincide in reflexive spaces.

The classical results in metric fixed point theory state that a Banach space has w-FPP if its norm is uniformly Fréchet differentiable $(\underline{\mathrm{K}})$ or uniformly rotund ([B] $)$. In fact, instead of uniformly rotund, it is sufficient to assume that the norm is only uniformly rotund in every direction (URED), [Z. It is a natural question whether the uniform Fréchet differentiability can be weakened to uniform Gâteaux differentiability (UG), since the notion of UG is dual (in a sense) to URED. (In fact, UG is dual to weak* uniform rotundity, which is a stronger notion than URED.)

We note that in a non-separable case, a theorem of [DLT] states that for any uncountable set $\Gamma$, the non-separable space $c_{0}(\Gamma)$ does not have FPP under any equivalent renorming. But it is well known that for any set $\Gamma, c_{0}(\Gamma)$ has an equivalent renorming that is simultaneously locally uniformly rotund, Fréchet differentiable and UG; see e.g. [DGZ, II.7.8]. Thus even norms with rather good geometrical properties do not assure FPP.

In our note we show that the usual proofs of "UF, UR or URED implies w-FPP" cannot be adapted, since they prove the w-FPP by showing that UF, UR or URED implies that the norm has a normal structure. We show that, in contrast, if the norm of a Banach space is UG, it does not necessarily have a normal structure. Even more, every separable Banach space can be equivalently renormed to have a uniformly Gâteaux smooth norm that lacks asymptotic normal structure. This

Received by the editors January 9, 2006.

2000 Mathematics Subject Classification. Primary 46B20.

Key words and phrases. Normal structure, asymptotic normal structure, uniformly Gâteaux smooth norms.

The first author was supported by the research project MSM 0021620839 and by grant GAČR $201 / 05 /$ P582.

The second author was supported by the UNCG New Faculty Summer Excellence Grant 2005. 1511

(C)2006 American Mathematical Society

Reverts to public domain 28 years from publication 
notion was defined by J. B. Baillon and R. Schöneberg in [BS] as a weakening of the normal structure, which is still sufficient for w-FPP.

The norm $\|\cdot\|$ on a Banach space $X$ is said to have asymptotic normal structure if for every closed convex bounded set $C \subset X$ with $\operatorname{diam} C>0$ and every sequence $\left\{x_{n}\right\} \subset C$ satisfying $\lim _{n \rightarrow \infty}\left\|x_{n}-x_{n+1}\right\|=0$ there exists $x \in C$ such that

$$
\liminf _{n \rightarrow \infty}\left\|x_{n}-x\right\|<\operatorname{diam}_{\|\cdot\|} C .
$$

The norm is called uniformly Gâteaux smooth if the limit

$$
\lim _{t \rightarrow 0} \frac{\|x+t h\|-\|x\|}{t}=\|\cdot\|^{\prime}(x, h)
$$

is uniform in $x \in S_{X}$ for each $h \in S_{X}$, where $S_{X}$ is the unit sphere of $X$. It follows that the derivative of the norm at $x \in X \backslash\{0\}$, i.e. $h \mapsto\|\cdot\|^{\prime}(x, h)$ is an element of $X^{*}$.

Recall that a Markushevich basis of a Banach space $X$ is a biorthogonal system $\left\{e_{n} ; f_{n}\right\} \subset X \times X^{*}$ such that $\overline{\operatorname{span}}\left\{e_{n}\right\}=X$ and $\left\{f_{n}\right\}$ separates the points of $X$ (i.e. for any $x \neq y \in X$ there is $n \in \mathbb{N}$ such that $f_{n}(x) \neq f_{n}(y)$ ).

Theorem 1. Let $X$ be a separable Banach space. Then there exists an equivalent uniformly Gâteaux smooth norm lacking asymptotic normal structure.

Proof. First, we will define a norm that lacks asymptotic normal structure. It will be done similarly as in $\mathrm{MS}$. Let $\left\{e_{n} ; f_{n}\right\}$ be a Markushevich basis of $(X,\|\cdot\|)$ such that $\left\|e_{n}\right\|=1$ and $\left\|f_{n}\right\| \leq 20$ for all $n \in \mathbb{N}$ (see e.g. [LT, 1.f.4]). We put

$$
C=\left\{x \in X ;\|x\| \leq 2,0 \leq f_{n}(x) \leq 1 \text { for all } n \in \mathbb{N}\right\} .
$$

This is a closed convex bounded set, $0 \in C$ and $\left\{e_{n}\right\} \subset C$. For an arbitrary $\beta \geq \operatorname{diam}_{\|\cdot\|} C$, we define a new norm

$$
\|x\|_{\beta}=\max \left\{\|x\|, \beta \sup _{n \in \mathbb{N}}\left|f_{n}(x)\right|\right\},
$$

which is obviously an equivalent norm on $X$.

Fact 2. For all $n \in \mathbb{N},\left\|e_{n}\right\|_{\beta}=\beta$ and $\left\|f_{n}\right\|_{\beta}^{*}=1 / \beta$.

Proof of Fact 2 .

$$
\left\|e_{n}\right\|_{\beta}=\max \left\{\left\|e_{n}\right\|, \beta \sup _{k \in \mathbb{N}}\left|f_{k}\left(e_{n}\right)\right|\right\}=\max \{1, \beta\}=\beta .
$$

Regarding $f_{n}$, we have

$$
\left\|f_{n}\right\|_{\beta}^{*} \geq f_{n}\left(\frac{e_{n}}{\beta}\right)=\frac{1}{\beta},
$$

and, on the other hand,

$$
\begin{aligned}
\left\|f_{n}\right\|_{\beta}^{*} & =\sup \left\{f_{n}\left(\frac{\sum_{k=1}^{N} a_{k} e_{k}}{\left\|\sum_{k=1}^{N} a_{k} e_{k}\right\|_{\beta}}\right) ; N \geq n, a_{1}, \ldots, a_{N} \in \mathbb{R}\right\} \\
& =\sup _{a_{n} \neq 0} \frac{a_{n}}{\left\|\sum_{k=1}^{N} a_{k} e_{k}\right\|_{\beta}} \leq \frac{a_{n}}{\beta a_{n}}=\frac{1}{\beta},
\end{aligned}
$$


where the inequality holds because, by the definition,

$$
\left\|\sum_{k=1}^{N} a_{k} e_{k}\right\|_{\beta} \geq \beta f_{n}\left(\sum_{k=1}^{N} a_{k} e_{k}\right)=\beta a_{n} .
$$

Fact 3. $\operatorname{diam}_{\|\cdot\|_{\beta}} C=\beta$.

Proof of Fact 3. First, $\operatorname{diam}_{\|\cdot\|_{\beta}} C \geq\left\|e_{1}-0\right\|_{\beta}=\beta$. On the other hand, if $x, y \in C$, then $\left(\right.$ as $\left.f_{n}(x), f_{n}(y) \in[0,1]\right)\left|f_{n}(x-y)\right| \leq 1$ and thus

$$
\|x-y\|_{\beta}=\max \left\{\|x-y\|, \beta \sup _{n \in \mathbb{N}}\left|f_{n}(x-y)\right|\right\} \leq \beta .
$$

Now we define a norm $\|\cdot \cdot\|_{\beta}^{*}$ on $X^{*}$ by a formula

$$
\left(\|\| f \|_{\beta}^{*}\right)^{2}=\left(\|f\|_{\beta}^{*}\right)^{2}+\sum_{n=1}^{\infty} \frac{1}{2^{n}} f^{2}\left(e_{n}\right) .
$$

By a standard convexity argument (see [DGZ, Fact II.2.3]), the norm $\|\cdot \cdot\|_{\beta}^{*}$ is $\mathrm{W}^{*} \mathrm{UR}$. Since \|\|$\cdot \|_{\beta}^{*}$ is weak*-lsc, it is a dual norm. Let $\|\cdot \cdot\|_{\beta}$ be the norm on $X$ that is predual to $\|\cdot\|_{\beta}^{*}$. By a standard duality argument (see DGZ, Thm. II.6.7]), the norm $\|\mid \cdot\|_{\beta}$ is uniformly Gâteaux smooth.

Fact 4. a) $\lim _{n \rightarrow \infty}\left|\left\|f_{n} \mid\right\|_{\beta}^{*}=1 / \beta\right.$,

b) $\lim _{n \rightarrow \infty}\left|\left\|e_{n} \mid\right\|_{\beta}=\beta\right.$,

c) $\operatorname{diam}_{\|\cdot\| \|_{\beta}} C=\beta$.

Proof of Fact 4, a) Follows directly from Fact 2

b) Since $\|f \mid\|_{\beta}^{*} \geq\|f\|_{\beta}^{*}$ for all $f \in X^{*}$, we have $\|x\|_{\beta} \leq\|x\|_{\beta}$ for all $x \in X$ and thus $\left\|e_{n} \mid\right\|_{\beta} \leq \beta$. On the other hand

$$
\liminf _{n \rightarrow \infty}\left\|e_{n}\right\| \|_{\beta} \geq \liminf _{n \rightarrow \infty} \frac{f_{n}\left(e_{n}\right)}{\left\|f_{n}\right\|_{\beta}^{*}}=\beta .
$$

c) As above, we get $\operatorname{diam}_{\|\cdot\| \|_{\beta}} C \leq \operatorname{diam}_{\|\cdot\|_{\beta}} C=\beta$. On the other hand,

$$
\operatorname{diam}_{\|\cdot\| \cdot \|_{\beta}} C \geq\left\|e_{n}\right\|_{\beta} \rightarrow \beta
$$

Now we are ready to prove that $\|\mid \cdot\|_{\beta}$ does not have asymptotic normal structure. Indeed, we define the sequence $\left\{x_{n}\right\} \subset C$ by

$$
x_{n}=\left\{\begin{array}{l}
\left(1-j 2^{-2 k}\right) e_{k}+e_{k+1}, \text { where } n=2^{2 k}+j, j=1, \ldots, 2^{2 k} \\
e_{k+1}+j 2^{-2 k-1} e_{k+2}, \text { for } n=2^{2 k+1}+j, j=1, \ldots, 2^{2 k+1}
\end{array}\right.
$$

Clearly, $x_{n} \in C$ and

$$
\lim _{n \rightarrow \infty}\|\| x_{n}-x_{n+1} \|_{\beta}=0
$$


Choose $x \in C$. For any $\varepsilon>0$ let $N \in \mathbb{N}$ and $y=\sum_{l=1}^{N} a_{l} e_{l}$ be such that $\|x-y\|_{\beta}<$ $\varepsilon$. Then, for all $k>N$ and all $n=2^{2 k+i}+j, j=1, \ldots, 2^{2 k+i}, i=0,1$,

$$
\left\|x-x_{n}\right\|_{\beta}>\left\|y-x_{n}\right\|_{\beta}-\varepsilon \geq \frac{f_{k+1}\left(y-x_{n}\right)}{\left\|f_{k+1}\right\|_{\beta}^{*}}-\varepsilon=\frac{1}{\left\|f_{k+1}\right\|_{\beta}^{*}}-\varepsilon .
$$

Thus,

$$
\beta \geq \liminf _{n \rightarrow \infty}\left\|x-x_{n}\right\|_{\beta} \geq \beta-\varepsilon,
$$

and consequently $\lim _{n \rightarrow \infty} \mid\left\|x-x_{n}\right\|_{\beta}=\beta=\operatorname{diam}_{\|\cdot\|} \|_{\beta} C$.

Remark. Note that in the proof we could take $C=\overline{\operatorname{conv}}\left\{0, e_{n}, e_{n}+e_{n+1} ; n \in \mathbb{N}\right\}$. If the basis $\left\{e_{n}\right\}$ is weakly null, then by Krein's theorem $C$ is weakly compact, and hence we have an example of a weakly compact convex set without asymptotic normal structure.

\section{ACKNOWLEDGMENTS}

The authors would like to thank the Texas A\&M University at College Station, Texas and Dr. W. B. Johnson in particular, for organizing the NSF supported Workshop in Linear Analysis and Probability, 2005, during which this project was initiated.

\section{REFERENCES}

[BS] J.B. Baillon and R. Schöneberg, Asymptotic normal structure and fixed points of nonexpansive mappings, Proc. Amer. Math. Soc. 81 (1981), no. 2, 257-264. MR0593469 (82c:47068)

[B] F.E. Browder, Nonexpansive nonlinear operators in a Banach space, Proc. Nat. Acad. Sci. U.S.A. 54 (1965), 1041-1044. MR0187120 (32:4574)

[DGZ] R. Deville, G. Godefroy and V. Zizler, Smoothness and Renormings in Banach Spaces, Monographs and Surveys in Pure and Applied Mathematics 64, Pitman, 1993. MR1211634 (94d:46012)

[DLT] P.N. Dowling, C.J. Lennard and B. Turett, Asymptotically isometric copies of $c_{0}$ in Banach spaces, J. Math. Anal. Appl. 219 (1998), 377-391. MR1606342 (98m:46023)

[K] M.A. Khamsi, Uniform smoothness implies super-normal structure property, Nonlinear Anal. 19 (1992), 1063-1069. MR.1194145 (93k:46012)

[M] B. Maurey, Points fixes des contractions sur un convexe fermé de $L_{1}$, Seminaire d'Analyse Fonctionelle 80-81, Ecole Polytechnique Palaiseau, 1981. MR0659309 (83h:47041)

[MS] S.A. Mariadoss and P.M. Soardi, A remark on asymptotic normal structure in Banach spaces, Rend. Sem. Mat. Univ. Politec. Torino 44 (1987), no. 3, 393-395. MR0932426 (89e:46017)

[LT] J. Lindenstrauss and L. Tzafriri, Classical Banach Spaces I. Sequence Spaces, SpringerVerlag, 1977. MR0500056 (58:17766)

[Z] V. Zizler, On some rotundity and smoothness properties of Banach spaces, Dissertationes Math. Rozprawy Mat. 87 (1971), 33 pp. MR0300060 (45:9108)

Department of Mathematical Analysis, Charles University, Sokolovská 83, 18675 Praha 8, Czech Republic

E-mail address: johanis@karlin.mff.cuni.cz

Department of Mathematical Sciences, University of North Carolina at Greensboro, Greensboro, North Carolina 27402

E-mail address: rychtar@uncg.edu 